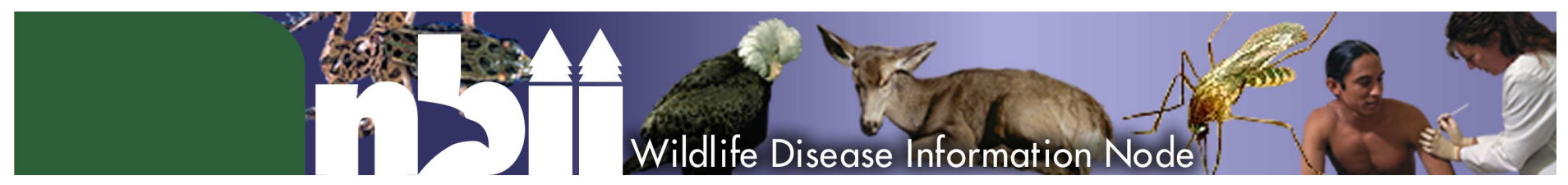

\title{
Highly Pathogenic Avian Influenza Early Detection Data System (HEDDS)
}

\section{HEDDS offers a unique opportunity for multiagency cooperation for data sharing and visualization.}

\section{Background on the NBII}

The National Biological Information Infrastructure (NBII) www.nbii.gov is an electronic information network that provides access to biological data and information on our nation's plants, animals, and ecosystems.

Information contributed by federal, state, and local government agencies; non-government organizations; and private-sector organizations is linked through the NBII gateway and made accessible to a variety of audiences, including researchers, natural resource managers, decision-makers, educators, students, and other members of the general public. Implementation of the NBII is being accomplished through the development of "nodes" that serve as entry points to the network. These nodes function as fully digital, distributed, and interactive systems that focus on content in a defined subject area or a geographic region. The NBII Wildlife Disease Information Node (WDIN) addresses the need for information on a variety of disease agents in wildlife and their implications, including those affecting domestic animals and people.

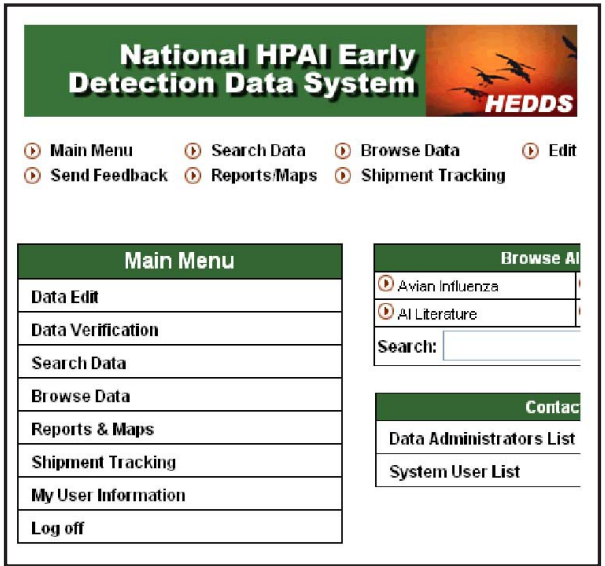

Main menu page. Data fields collected include: species, sex, age, geographic location, sample type, test requested, test results (+1- for Highly Pathogenic H5N1 only).

\section{Background on HEDDS}

Avian influenza (AI) is a type A influenza virus naturally found in certain species of waterfowl and shorebirds. The occurrence of highly pathogenic avian influenza (HPAI) subtype H5N1 circulating in Southeast Asia has raised concerns regarding its potential impact on wild birds, domestic poultry, and human health, should it be introduced into the United States.

At the request of the White House Policy Coordinating Committee for Pandemic Influenza Preparedness, the U.S. Departments of Agriculture and Interior, along with other partners, developed an early detection plan for HPAI in the United States. The plan calls for the establishment of a national database for use by all agencies, organizations and policy makers. WDIN, housed at the U.S. Geological Survey's (USGS) National Wildlife Health Center, has created the HPAI
Early Detection Data System, http://wildlifedisease.nbii.gov/ai, to meet this goal. An Interagency Steering Committee (ISC) has been established to direct the operation and content of the system.

\section{HEDDS Data Collection}

HEDDS was developed to manage animal and specimen collection data taken by many groups and individuals, and analyzed by multiple laboratories.

It provides a secure, accessible platform for the generation of reports, graphs, and maps and can be used for spatial modeling.

The U.S. Interagency Strategic Plan for Early Detection of Asian H5N1 Highly Pathogenic Avian Influenza in Wild Migratory Birds describes five surveillance strategies:

- Investigation of morbidity and mortality events,

- Surveillance of live wild birds,

- Surveillance of hunter-killed birds,

- Surveillance of sentinel species, and

- Environmental sampling.

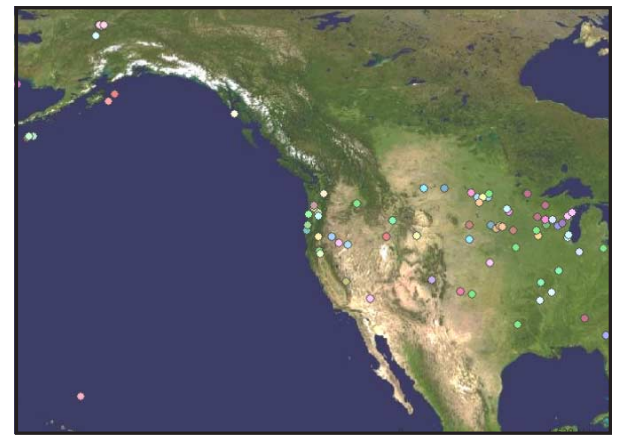

Map created by HEDDS showing sites where specimens have been collected. 
HEDDS is a versatile system that can hold data from these surveillance strategies as well as accommodate future surveillance plans, and it can provide a critical comprehensive view of national sampling efforts.

Managing data includes ensuring the resulting data are available for viewing and analysis by all contributors. For this purpose, incorporating appropriate standards into the data system is essential to facilitate information sharing and to improve surveillance strategic planning.

\section{System Interface and Functions}

\section{Access and Security}

The system is password protected. Each contributing agency or organization agrees to share summaries from the core data fields, as selected by the ISC. These fields and data elements have been developed to comply with existing standards for the transmission of electronic health information. While HEDDS is a collaborative system, through ISC discussions, contributors determine who has access to their data records.

\section{Data Viewing and Entry}

Data may be browsed in their entirety, or filtered by various parameters (e.g., species, sex, location). Standardized reports for individual contributors, as well as customized report options are available. The core data fields are used to construct standardized summaries of results in the database (e.g., number of specimens tabulated by location). Active contributors will define publicly viewable reports and maps.

Data can be entered using Web entry forms or through uploading an MS Excel spreadsheet, which converts data to the required HEDDS data format. A tracking function is available to monitor sample shipments, and thereby enhances coordination between diagnostic laboratories and field personnel who are collecting the samples.

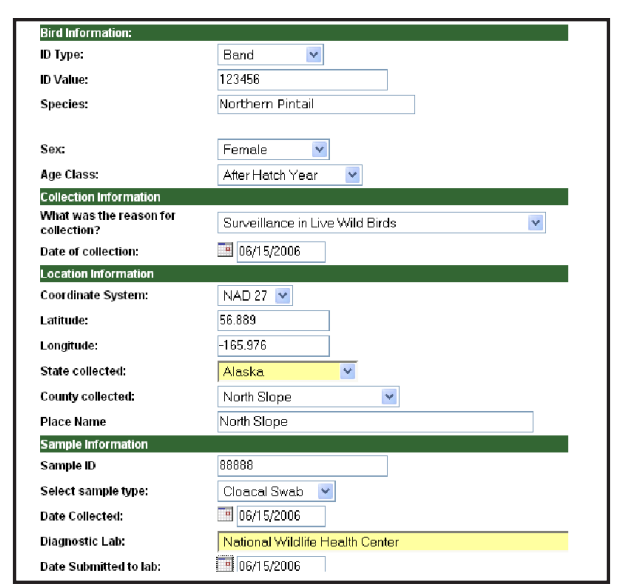

Data entry form. All data fields and elements link to established standards, consistent with those of the National Animal Health Laboratory Network (NAHLN).

\section{Mapping Capabilities}

In addition to using the database to generate generic or customized AI specimen/testing reports, the system provides on-demand maps, with customizable data fields, overlain on a wide range of layers. These layers are made possible by the utilization of the NBII Geospatial Information Framework toolset.

This framework is an implementation of Open GIS Standards and allows users to incorporate layers from external sources. Users can search and add a wide variety of background and foreground layers including USGS Topographic maps, Digital Orthophotos, road networks, bodies of water, and species population distribution.

\section{Connecting to the WDIN Avian Influenza Library}

While HPAI data, maps, and reports provide critical insight to avian influenza sampling efforts, access to the diverse body of influenza knowledge is equally as valuable to surveillance projects and to the understanding of this disease. From the HEDDS system, users can access authoritative avian influenza Web resources on the WDIN Web site. This organized and searchable resource guide contains an AI bibliography (over 225 articles), maps, news sources and selected Web site linkages.

\section{Future}

HEDDS offers a unique opportunity for multiagency cooperation for data sharing and visualization. The system will evolve as necessary to meet contributor needs and integrate data from new contributors engaged in avian influenza surveillance and research efforts.

\section{For More Information}

Robert Worrest

WDIN Node Manager

USGS Biological Informatics Office

NBII Program

Phone: 703-648-4074

E-mail: rworrest@usgs.gov

F. Joshua Dein

Project Leader

USGS National Wildlife

Health Center

Phone: 608-270-2450

E-mail: joshua_dein@usgs.gov

Find us on the Web at http://

wildlifedisease.nbii.gov/ai.

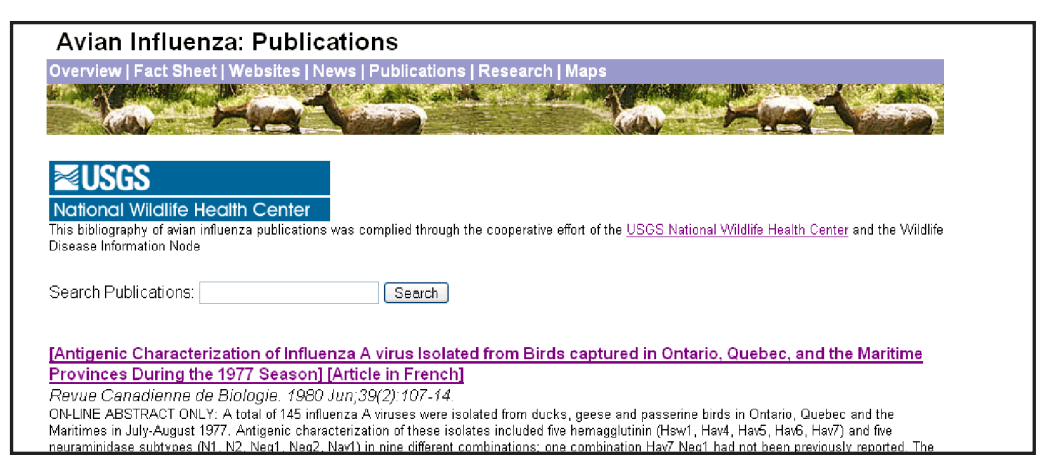

Avian Influenza publications are searchable on the WDIN Web site http://wildlifedisease.nbii.gov/. 